Annls Limnol. 16 (1) $1980: 21-41$.

\title{
COEFFICIENTS THERMIQUES ET ECOLOGIE DE QUELQUES PLANAIRES D'EAU DOUCE VII : LEUR ZONATION NATURELLE
}

\author{
par E. PATTEE 1
}

La carte altitudinale et l'enregistrement des moyennes thermiques trimestrielles pendant 3 ans dans 35 stations de la région lyonnaise fournissent la même image de la distribution des Planaires: les espèces rhéophilles $C$. alpina, $P$. felina et $D$. gonocephala occupent trois zones sucessives avec des recouvrements de seulement 0,3 à $0,5^{\circ} \mathrm{C}$ dans leurs températures annuelles moyennes. La zone des espèces limnophiles $P$. tenuis et $P$. nigra double, en eau calme, les deux zones de $P$. felina et $D$. gonocephala. Enfin $D$. tigrina semble s'étendre davantage vers les températures élevées.

Sans qu'il y ait concordance des valeurs absolues, cette sucession reproduit celle qui apparaît dans la physiologie des mêmes espèces, laissant à penser que les mécanismes de la zonation sont d'ordre compétitif. Le remplacement de $D$. gonocephala par la souche de $P$. felina introduite dans une station située au niveau du secteur de recouvrement de leurs deux zones thermiques, confirme cette hypothèse.

\section{The temperature relations of some fresh-water planarians and their incidence in ecology.}

\section{Their natural zonation.}

The sucrose inversion method was applied to determine the mean temperature over successive periods of 3 months in 35 stations inhabited by planarian triclads in the Lyons region. Most investigations were carried out over a period of 3 years. The altitudinal distribution and the thermal range of these species appear to correspond. The three rheophilic triclads, $C$. alpina, $P$. felina, and $D$. gonocephala occupy three successive zones with only 0.3 to $0.5^{\circ} \mathrm{C}$ overlap in their annual mean temperature. The zone of the limnophilic $P$. tenuis and $P$. nigra covers, in still water, that of $P$. felina and $D$. gonocephala. D. tigrina seems to favour warmer temperatures.

Though the absolute values do not coincide, this pattern reproduces the picture given by physiological investigations, hinting at the competitive nature of most of the mechanisms which determine the distribution of these animals.

In one station, $D$. gonocephala was shown to live at the lower end of its range, in the zone of thermal overlap with that of P. felina. When the latter was introduced, it totally replaced the former within 3 years. This confirms our hypothesis.

D'ordre expérimental, nos précédentes investigations sur les cœfficients thermiques chez les Planaires (Pattee 1966 à Russier-Delolme 1972) ont permis de classer les espèces en une série continue, en fonc-

1. Département de Biologie animale et Zoologie, Université Cl.-Bernard, 43, bd di 11-Novembre, F-69622 Villeurbanne Cedex. 
tion de leurs réactions à la température. Simultanément étaient relevées les températures qui règnent, sur le terrain, dans les divers secteurs habités par les mêmes espèces. Décrits ici, ces derniers résultats devraient permettre de comparer les exigences et les optimums physiologiques mesurés au laboratoire avec les conditions thermiques naturelles. Le degré de concordance des valeurs trouvées de part et d'autre pourra être considéré comme une indication du rôle effectivement joué par la température dans la distribution de ces espèces.

\section{1. - LA ZONATION THERMIQUE DES ESPECES ANIMALES EN GENERAL ET DES PLANAIRES EN PARTICULIER, D'APRES LES TRAVAUX ANTERIEURS}

En accord avec Macan 1956 et 1961, nombreux sont les auteurs qui considèrent la température comme l'un des facteurs les plus importants de la répartition des organismes aquatiques. Il en est ainsi dans la comparaison entre milieux topographiquement voisins, mais de faciès différent, et particulièrement dans celle des deux domaines lotique et lénitique (Despax 1927, Whitney 1939, Park 1945, Walshe 1948, Roux et Roux 1967, Spence et Hynes 1971, Ward 1974, Lascombe 1974, Pattee 1969 et d'autres. La température intervient manifestement de façon prépondérante dans la zonation latitudinale des espèces et des races aquatiques : Brown 1929, Moore 1939 et 1949, Scholander, Flagg, Walters et Irving 1953, Tashian 1956, Roberts 1957, Vernberg et Tashian 1959, Vernberg 1959 a et b mettent en évidence des adaptations physiologiques ou éthologiques liées à la température plus basse ou plus élevée de l'habitat.

La zonation altitudinale et la zonation amont-aval le long des cours d'eau coïncident avec un gradient thermique susceptible d'expliquer le remplacement progressif des espèces les unes par les autres. Des exemples démonstratifs en sont fournis par Dodds et Hisaw 1925, Pleskot 1951, Kamler 1965, Philipson et Moorhouse 1976. Huet (1949) reconnaît l'influence de la température dans la règle des pentes bien connue, qui lui permet de caractériser les zones piscicoles. De façon plus précise, Edington et Hildrew (1973) trouvent deux peuplements de Trichoptères spécifiquement et physiologiquement différents selon qu'ils proviennent de rivières à température estivale supérieure ou inférieure à $15^{\circ} \mathrm{C}$. Pour Décamps (1967 et 1968) l'étagement du même groupe zoologique en altitude, dans les ruisseaux des Pyrénées, paraît liée à l'amplitude thermique annuelle et donc plus spécialement au maximum relevé en juillet-août.

Verneaux (1976) obtient, par analyse factorielle des correspondan- 
ces à partir de la faune récoltée, une classification en zones biotypologiques à l'intérieur de l'écosystème « eau courante". Il relève par ailleurs (1977) différents paramètres morphodynamiques et physicochimiques, dont la température maximale moyenne du mois le plus chaud (juillet). L'analyse factorielle fait alors apparaître la température du milieu, évaluée par cet indice simple, comme l'un des facteurs essentiels de la succession des zones biotypologiques.

Considérant la faune benthique des Wiedemannia, des Rhyacophila ct des Planaires Triclades, Vaillant avait auparavant été amené en 1967 a et b et en 1972, à définir 7 zones successives dans les eaux courantes alpestres, zones qu'il caractérisait par leurs espèces indicatrices et qu'il considérait, sans pouvoir le démontrer, comme déterminées par la somme totale annuelle des températures qui y règnent, notion sur laquelle nous reviendrons.

En fait, les Planaires sont l'un des groupes dans l'écologie desquels la température intervient de la façon la plus évidente, ne serait-ce que par la présence simultanée de Crenobia alpina dans les Alpes et en Scandinavie. A la suite de Voigt (1895) et Steinmann (1907), nombreux sont les auteurs qui ont relevé la succession altitudinale plus ou moins régulière des 3 espèces rhéophiles Crenobia alpina, Polycelis Jelina et Dugesia gonocephala, après lesquelles apparaît souvent lorsque les conditions d'agitation de l'eau le permettent, la limnophile Poiycelis nigra.

La zonation des espèces de Planaires semble d'ailleurs être un phénomène assez général pour avoir été constatée ailleurs qu'en Europe, sur des formes différentes. En Amérique, dans l'Illinois, Chandler (1965) retrouve cette répartition amont-aval dans une rivière, entre Cura foremani et Dugesia tigrina, et dans une autre entre Phagocata gracilis et Dugesia dorotocephala. La zonation altitudinale remarquable des 5 espèces japonaises a été étudiée en détail par Kawakatsu (1965, 1970 et 1974), Kawakatsu, Nimura et Horikoshi (1974) et attribuée en grande partie au régime thermique des milieux considérés.

Le tableau I donne une liste des principaux auteurs qui ont constaté l'étagement des espèces européennes et qui fournissent des relevés de température dans l'un des niveaux considérés. Beaucoup des relevés sont ponctuels et sans indication sur l'heure de la journée à laquelle ils sont effectués. Certains font état des valeurs extrêmes notées au cours de l'année et parfois d'une moyenne, mais les bases de calcul de cette dernière sont rarement précisées.

Seuls Vandel (1919) et Ball (1964) concluent à une nette absence de zonation thermique dans les situations qu'ils étudient. Mais malgré l'avis presque unanime des autres auteurs, cette zonation ne ressort pas nettement des chiffres du tableau, par suite de la présentation hétérogène des données, et sans doute aussi pour la raison mise en 
Tableau I. - La température des milieux abritant les diverses espèces de Planaires étu. diées ici : quelques données de la littérature. Sauf indication contraire, $P$. nigra et $P$. tenuis sont traitées ensemble, de nombreux auteurs ne les distinguant pas l'une de l'autre.

\begin{tabular}{|c|c|c|}
\hline Auteur & Lieu & Températures \\
\hline \multicolumn{3}{|l|}{ Crenobia alpina } \\
\hline Steinmann 1907 a & Alpes, Jura, Forêt Noire & Maturité 4,5 à $9^{\circ} \mathrm{C}$. Optimum $5-6^{\circ} \mathrm{C}$ \\
\hline Sieinböck 1942 a & $\begin{array}{l}\text { Alpes : ruisseaux et } \\
\text { flaques }\end{array}$ & $\begin{array}{l}\text { Maximum passager } 22^{\circ} \mathrm{C} \\
\text { Amplitude journalière }>17^{\circ} \mathrm{C}\end{array}$ \\
\hline Pleskot 1951 & Alpes autrichiennes & Annuellement 1,5 à $17-19^{\circ} \mathrm{C}$ \\
\hline Vaillant $1967 \mathrm{~b}$ & $\begin{array}{l}\text { Alpes françaises } \\
\text { Zones } \mathrm{O}-\mathrm{A}-\mathrm{B}>1000 \mathrm{~m}\end{array}$ & $<15^{\circ} \mathrm{C}$ \\
\hline Steinböck 1957 & Lac Majeur & $<13,5^{\circ} \mathrm{C}$ annuellement \\
\hline Seintböck 1942 b & Scandinavie & $1.3^{\circ} \mathrm{C}$ \\
\hline Dahm 1958 & Suède & $\leqslant 14^{\circ} \mathrm{C}$ \\
\hline Luther 1961 & \multicolumn{2}{|c|}{$\begin{array}{l}\text { Finlande } \\
\text { Seulement au Nord du Cercle Polaire }\end{array}$} \\
\hline Beauchamp et Ullyott 1932 & Balkans & $<16-17^{\circ} \mathrm{C}$. En compétition $<13^{\circ} \mathrm{C}$ \\
\hline Stankovic 1935 & Balkans $>600 \mathrm{~m}$ & $\begin{array}{l}3 \text { à } 17^{\circ} \mathrm{C} \text {, moyenne } 8-9^{\circ} \mathrm{C} \text {, forme } \\
\text { montenigrina }\end{array}$ \\
\hline Vandel 1919 & Jura & $\begin{array}{l}0-20^{\circ} \mathrm{C} \text { annuellement, aucune zonation } \\
\text { thermique }\end{array}$ \\
\hline Pattee 1965 & Jura, suintement & $\leqslant 25^{\circ} \mathrm{C}$. Amplitude journalière $15^{\circ} \mathrm{C}$ \\
\hline Thienemann 1913 & Sauerland & $\begin{array}{l}\text { Annuellement } 3 \text { à } 13{ }^{\circ} \mathrm{C} \\
\text { moyenne } 7,1 \text { à } 7,6^{\circ} \mathrm{C}\end{array}$ \\
\hline Schlieper et Bläsing 1952 & $\begin{array}{l}\text { Marburg a.d.Lahn, } \\
\text { source }\end{array}$ & $\begin{array}{l}8,9 \text { à } 10,2^{\circ} \mathrm{C} \\
\text { généralement }<11^{\circ} \mathrm{C}\end{array}$ \\
\hline Becker 1960 & Württemberg & $\begin{array}{l}\text { généralement } 5 \text { à } 15^{\circ} \mathrm{C} \\
\text { rarement } 15-20^{\circ} \mathrm{C}\end{array}$ \\
\hline Flössner 1963 & Erzgebirge & $<20^{\circ}$. Température estivale $9-15^{\circ} \mathrm{C}$ \\
\hline Giesen-Hildebrand 1975 & $\begin{array}{l}\text { Siebengebirge } \\
\text { Sources et ruisseaux }\end{array}$ & 4 à $17,5^{\circ} \mathrm{C}$ annuellement \\
\hline Mercier 1909 & Nancy, source & 9,5 à $10^{\circ} \mathrm{C}$ toute l'année \\
\hline Oye 1935 in Becker 1960 & Luxembourg & généralement $<11^{\circ} \mathrm{C}$ \\
\hline Oye 1941 & Belgique & températures basses \\
\hline Leloup 1944 & $\begin{array}{l}\text { Forêt de Soignes (Belgi- } \\
\text { que), altitude } 70 \mathrm{~m}\end{array}$ & 1,5 à $20,8^{\circ} \mathrm{C}$ annuellement \\
\hline Carpenter 1928 & $\begin{array}{l}\text { Cardiganshire } \\
\text { altitude }<300 \mathrm{~m}\end{array}$ & $\begin{array}{l}<19,5^{\circ} \mathrm{C} \text {. Maturité à } 11,5^{\circ} \mathrm{C} \\
\text { Migrations saisonnières }\end{array}$ \\
\hline Ball 1964 & Hertforshire, sources & $\begin{array}{l}9,8 \text { à } 10,2^{\circ} \mathrm{C} \text {. Aucune zonation ther- } \\
\text { mique }\end{array}$ \\
\hline Wright 1972 & $\begin{array}{l}\text { Caernarvonshire } \\
0 \text { à } 795 \mathrm{~m} \\
\text { Anglesey sources } \\
0 \text { à } 150 \mathrm{~m}\end{array}$ & $\begin{array}{l}6,9 \text { à } 11,8^{\circ} \mathrm{C} \\
6,5 \text { à } 14^{\circ} \mathrm{C}\end{array}$ \\
\hline Arndt 1922 & $\begin{array}{l}\text { Corse (sources) } \\
\text { Sibérie }\end{array}$ & $\begin{array}{l}5 \text { à } 14^{\circ} \mathrm{C} \\
8 \text { à } 13^{\circ} \mathrm{C} \text {, exceptionnellement } 20^{\circ} \mathrm{C}\end{array}$ \\
\hline
\end{tabular}




\section{Polycelis felina}

\begin{tabular}{|c|c|c|}
\hline Pleskot 1951 & Alpes autrichiennes & 0 à $17-19^{\circ} \mathrm{C}$ \\
\hline Vaillant 1967 b & $\begin{array}{l}\mathrm{S} \text { et } \mathrm{SE} \text { de la France } \\
\text { Zones BCDE }<1500 \mathrm{~m}\end{array}$ & Température estivale $\geqslant 15^{\circ} \mathrm{C}$. \\
\hline Beauchamp et Ullyott 1932 & Balkans & 10 à $17^{\circ} \mathrm{C}$ \\
\hline Stankovic 1935 & Balkans & 6,5 à $16,5^{\circ} \mathrm{C}$, moyenne $11,2^{\circ} \mathrm{C}$ \\
\hline Thienemann 1913 & Sauerland & $\begin{array}{l}\text { Annuellement } 0,5 \text { à } 15,7{ }^{\circ} \mathrm{C} \\
\text { moyenne } 7,3 \text { à } 7,6^{\circ} \mathrm{C}\end{array}$ \\
\hline Becker 1960 & Württemberg & 4,5 à $21,6^{\circ} \mathrm{C}$ \\
\hline Flössner 1963 & Erzgebirge & Température estivale $13-15^{\circ} \mathrm{C}$ \\
\hline Oye 1935 in Becker 1960 & Luxembourg & 0,5 à $15,7^{\circ} \mathrm{C}$ \\
\hline Oye 1941 & Belgique & Températures basses \\
\hline Leloup 1944 & $\begin{array}{l}\text { Forêt de Soignes (Belgi- } \\
\text { que) }\end{array}$ & 1,5 à $20,8^{\circ} \mathrm{C}$ \\
\hline Carpenter 1928 & Cardiganshire & $<17^{\circ} \mathrm{C}$ \\
\hline Ball 1964 & $\begin{array}{l}\text { Hertfordshire, sources } \\
\text { et ruisseaux }\end{array}$ & Aucune zonation thermique \\
\hline Wright 1972 & $\begin{array}{l}\text { Caernarvonshire } \\
<300 \mathrm{~m} \\
\text { Anglesey } 0 \text { 'à } 150 \mathrm{~m}\end{array}$ & $\begin{array}{l}7,7 \text { à } 10,3^{\circ} \mathrm{C} \text { annuellement } \\
3,8 \text { à } 15^{\circ} \mathrm{C} \text { annuellement }\end{array}$ \\
\hline Alause 1968 & Languedoc & 11 à $18^{\circ} \mathrm{C}$, rarement $22^{\circ} \mathrm{C}$ \\
\hline Légier 1969 & Provence & 7 à $17^{\circ} \mathrm{C}$ \\
\hline
\end{tabular}

\section{Dugesia gonocephala}

Pleskot 1951

Beauchamp et Ullyott 1932

Stankovic 1935

Steinmann 1907 a

Vandel 1919

Thienemann 1913

Becker 1960

Flössner 1963

Giesen-Hildebrand 1975

Mercier 1909

Oye 1935 in Becker 1960

Oye 1941

Leloup 1944

Madsen 1963

Arndt 1922

Alause 1968

Légier 1969
Alpes autrichiennes

Balkans

Balkans $<900 \mathrm{~m}$

Jura, Forêt Noire

Jura

Sauerland

Württemberg

Erzgebirge

Siebengebirge, ruisseau

Nancy

Luxembourg

Belgique

Forêt de Soignes (Belgique)

Jutland

Corse, ruisseaux

Languedoc

Provence
0 à $20-29^{\circ} \mathrm{C}$

8,5 à $23^{\circ} \mathrm{C}$; en compétition $>13^{\circ} \mathrm{C}$

8 à $27^{\circ} \mathrm{C}$, moyenne $12,9^{\circ} \mathrm{C}$

Maturité $13-17^{\circ} \mathrm{C}$

Aucune zonation thermique

annuellement 0,5 à $24^{\circ} \mathrm{C}$ moyenne estivale 11 à $16^{\circ} \mathrm{C}$

2,3 à $27,4^{\circ} \mathrm{C}$. Reproduction 7,5 à $12,6^{\circ} \mathrm{C}$

Zone commune avec $P$. felina et en aval

3 à $18,5^{\circ} \mathrm{C}$ annuellement

En aval de $C$. alpina

0 à $20^{\circ} \mathrm{C}$

Plus eurytherme que $C$. alpina et $P$. felina

1 à $20^{\circ} \mathrm{C}$, souvent avec $C$. alpina et P. felina

$\leqslant 16,5^{\circ} \mathrm{C}$

7,5 à $27,5^{\circ} \mathrm{C}$

9 à $22^{\circ} \mathrm{C}$

1 à $19^{\circ} \mathrm{C}$ 
Polycelis nigra et $\mathbf{P}$. tenuis

Vaillant 1967 b

Lascombe 1974

Becker 1960

Oye 1935 in Becker 1960

Oye 1941

Reynoldson 1960

Wright 1972

Luther 1961

Légier 1969

\section{Dugesia tigrina}

Russier et Lascombe 1970

Dahm 1958

Alause 1968

Légier 1969 et 1972

Chandler 1965
France centrale

Zone F

$\boldsymbol{P}$. tenuis

Région lyonnaise

Württemberg, en plaine

Luxembourg

Belgique

P. tenuis

Pays de Galles

P. nigra

Pays de Galles

P. tenuis

$S$ de la Finlande

Provence en aval de $D$. gonocephala

Moyenne estivale $\leqslant 22^{\circ} \mathrm{C}$

2 à $26^{\circ} \mathrm{C}$, en aval des 3 espèces rhéophiles

9 à $21,3^{\circ} \mathrm{C}$

Indifférente à la température

Température estivale 15 à $18^{\circ} \mathrm{C}$

2 à $17^{\circ} \mathrm{C}$

0 à $26^{\circ} \mathrm{C}$

3 à $20^{\circ} \mathrm{C}$

Région lyonnaise
Suisse, Angleterre
Languedoc
Provence
Indiana. USA

Région lyonnaise

1.5 à $23^{\circ} \mathrm{C}$

9 à $25^{\circ} \mathrm{C}$

7 à $23{ }^{\circ} \mathrm{C}$

2,5 à $20^{\circ} \mathrm{C}$ 0 à $32^{\circ} \mathrm{C}$. Moyennes hivernale $3^{\circ} \mathrm{C}$,
estivale $21^{\circ} \mathrm{C}$

avant par Steinböck (1942 a) et Pattee (1969 b) : occupant l'amont du réseau fluvial, Crenobia alpina habite souvent des milieux de petites dimensions et de très faible profondeur, donc très soumis aux variations atmosphériqus. Les maximums journaliers élevés, certains jours d'été, tendent, de ce fait, à masquer les moyennes basses et la diffélence avec les espèces suivantes. D'où la nécessité d'un bilan thermique plus complet, dans le sens des idées de Vaillant, dépassant ie stade des relevés ponctuels et même celui des maximums annuels utilisés par Verneaux.

L'approche écophysiologique et l'étude expérimentale comparée des réactions des diverses espèces de Planaires à la température conduisent à des constatations analogues. A la suite de Steinmann (1907 a) nombreux sont les auteurs, tels Steinböck ,(1942 a et 1951), Schlieper et Bläsing (1952), Becker (1960), qui qualifient ces réactions de degrés dans la sténothermie ou dans l'eurythermie. Les études écophysiologiques conduites dans notre laboratoire à la fois sur les limites létales, sur le métabolisme respiratoire et sur les paramètres de la reproduction et du développement, ont fait, de la part de Pattee, Lascombe et Delolme, l'objet d'un bilan en 1973. Ce dernier conduit à distinguer, 
à la suite d'Alause (1968), les deux notions d'amplitude thermique (liée à celle de sténothermie et d'eurythermie) et de niveau thermique moyen (liée à celle de psychrophilie et de thermophilie). Sans aller, comme l'auteur cité, jusqu'à nous poser la question de savoir si les premières sont "bonnes à pendre au musée des horreurs", nous attribuons aussi, dans le cas des Planaires concernées, le rôle écologique majeur aux secondes. Ainsi se classent par thermophilie croissante, mesurée expérimentalement, Crenobia alpina, Polycelis felina, Dugesia gonocephala, toutes trois rhéophiles, puis Polycelis nigra et P. tenuis, enfin Dugesia tigrina, plus limnophiles (Pattee et al., 1973).

\section{2. - STATIONS ET METHODE}

Les stations ont été choisies d'après les 3 critères suivants :

- Population autant que possible monospécifique et abondante.

- Accessibilité en toutes saisons à partir du laboratoire situé à Lyon. Ce critère exclut notamment la haute montagne, où Crenobia alpina est encore présente jusqu'à plus de $2500 \mathrm{~m}$ d'altitude.

- Sauf pour $P$. nigra et $D$. tigrina, espèces moins répandues, échantillonnage le plus vaste et le plus divers possible des faciès et des altitudes colonisés par chacune.

Les références relatives à ces stations figurent dans le tableau II.

La température a été relevée grâce à la méthode de l'inversion du saccharose, décrite par ailleurs (Pattee 1978). Son principe consiste à mesurer, par l'intermédiaire de cette réaction chimique, la moyenne des températures, stables ou variables, qui ont régné pendant un temps donné : les valeurs obtenues intègrent les températures de tous les instants, selon leur durée. Cependant la réaction étant commandée par la loi de Van't Hoff, la relation n'est pas linéaire mais exponentielle : les températures élevées ont plus de poids que les températures basses; les moyennes ne sont pas simplement des moyennes arithmétiques, mais d'autant supérieures à ces dernières que l'amplitude des variations est plus grande.

Avec Berthet 1960, on peut constater que les phénomènes biologiques sont gouvernés par des relations du même ordre, qualifier d' « écogiques " les moyennes mesurées et donc les considérer comme particulièrement adaptées à la présente étude.

Dans les stations, les flacons de sirop de saccharose étaient remplacés chaque trimestre pendant la période 1971-1973. Les pertes de flacons ont d'abord varié selon les stations et la période de l'année. Tenant compte de ces précédents, des flacons en plus grand nom- 


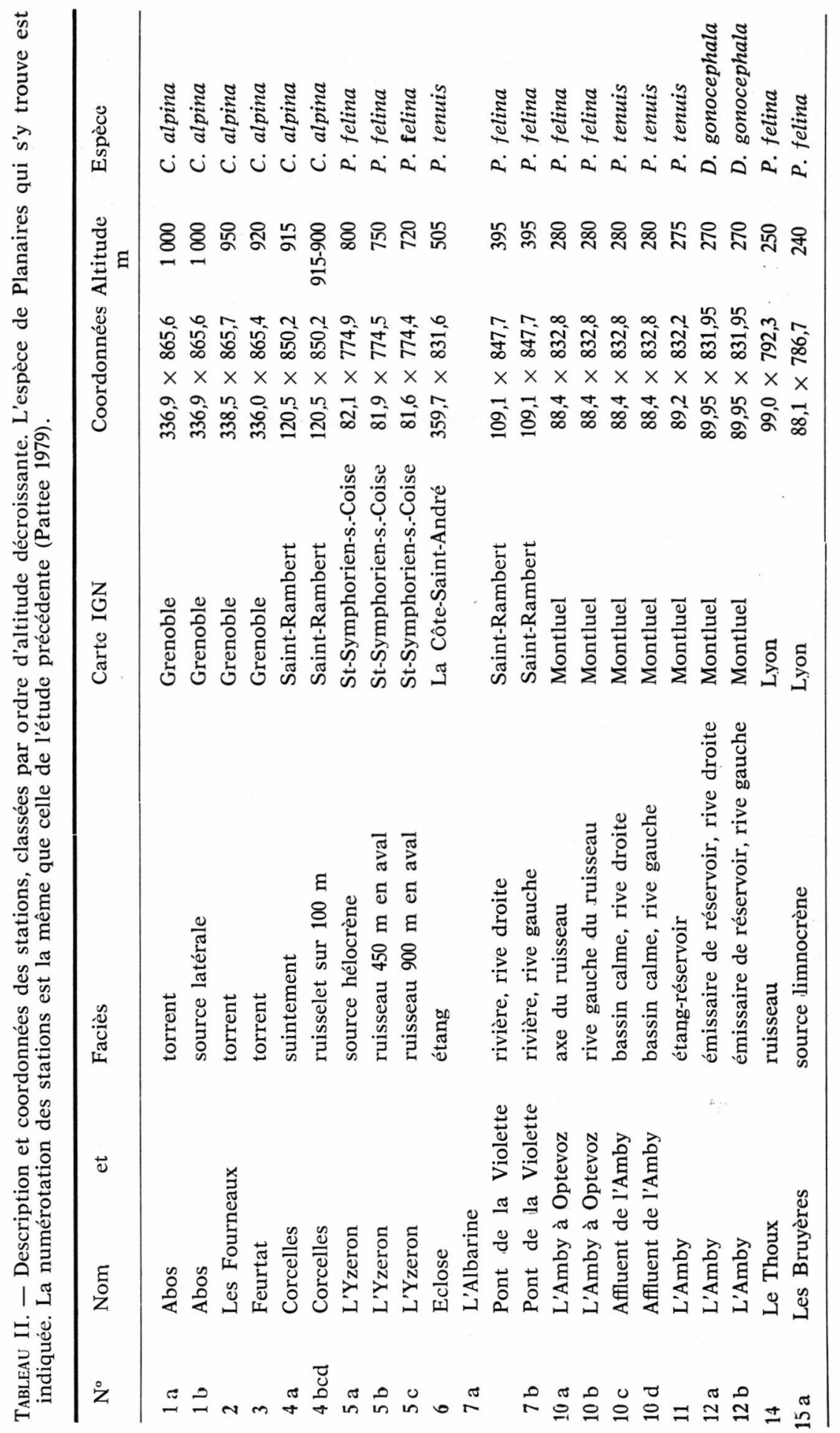




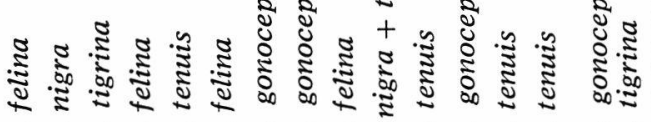

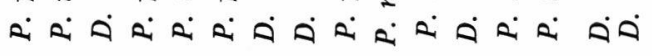

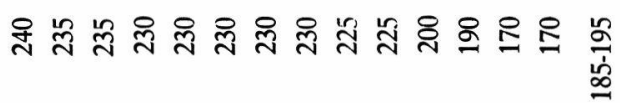

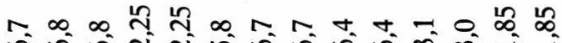

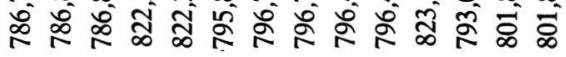
$\times \times \times \times \times \times \times \times \times \times \times \times \times \times$ के

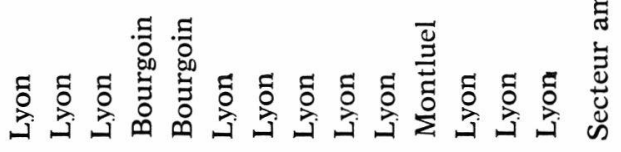

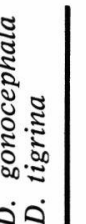$$
\stackrel{\longleftarrow}{\frac{1}{8}}
$$

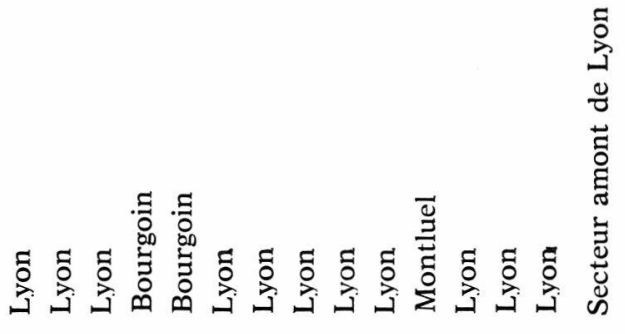

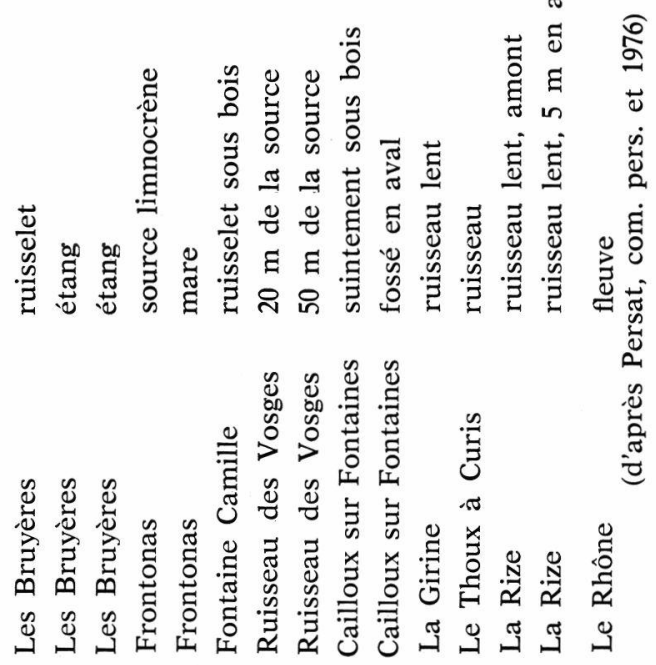

는 
bre ont été déposés dans les stations difficiles, c'est-à-dire celles à substrat instable, à niveau d'eau très variable ou prospectées par les pêcheurs ou baigneurs. De ce fait, seule l'année 1973 a donné des résultats complets dans toutes les stations. L'année 1974 a été mise à profit pour compléter les mesures aux emplacements et aux saisons où ces mesures etaient en nombre insuffisant. L'ensemble des valeurs trimestrielles pour les 4 saisons de ces années, recueillies dans les 36 stations, sont au nombre de plus de 400 .

Elles ont servi au calcul des moyennes thermiques annuelles, moyennes arithmétiques d'une part, moyennes « exponentielles » d'autre part, ces dernières obtenues par la formule (3) de Pattee (1978) et équivalentes aux valeurs qu'auraient donné des flacons de sirop exposés pendant l'année entière. Les résultats seront présentés :

- pour l'année 1973 seule: ils sont rigoureusement comparables ci'unc espèce à l'autre, mais décrivent la situation pour la seule année en cause, dont on sait qu'elle a eu un été plus chaud et un hiver plus froid que l'année precédente (Pattee 1979).

- pour l'ensemble des 4 années envisagées. Il est d'abord établi une movenne par saison (sur 2, 3 ou 4 années en fonction des lacunes de certains relevés) puis une moyenne annuelle à partir des 4 valeurs saisonnières obtenues. Quoique d'établissement moins rigoureux que la précédente par suite des quelques données manquantes, cette moyenne d'ensemble tend à éliminer les variations d'une année à l'autre, dont on sait qu'elles peuvent atteindre 1 à $2{ }^{\circ} \mathrm{C}$ dans l'eau (Pattee 1979). Elle se rapproche du niveau thermique absolu propre à l'espèce considérée.

\section{3. - RESULTATS}

\subsection{La zonation altitudinale}

Bien que représentant seulement un échantillon limité, la succession des stations dans le tableau II peut servir, avec quelques observations complémentaires, à contrôler l'étagement des espèces dans la région prospectée.

Sachant que, dans cette région, le niveau de base est celui du Rhône à $160 \mathrm{~m}$ d'altitude environ, le tableau permet les constatations suivantes :

- a. C. alpina règne sans concurrence au-dessus de $800 \mathrm{~m}$.

- b. P. felina lui succède, jusque vers $200 \mathrm{~m}$.

- c. La troisième espèce rhéophile, $D$. gonocephala apparaît dans la partie inférieure de l'étage à $P$. felina et jusqu'au niveau de base 
où ses races sexuée et surtout asexuée sont présentes en grand nombre dans le Rhône.

- d. Le domaine des Polycelis limnophiles, $P$. nigra et $P$. tenuis, semble doubler, en eau calme, les deux zones précédentes. Tirées de la liste donnée par Lascombe (1974), les stations à $P$. tenuis du tableau II montrent bien la grande extension de cette espèce et recouvrent la gamme des altitudes mentionnées par cet auteur dans la région lyonnaise. Dans le Jura et en Auvergne, l'espèce s'élève, selon la même source, jusqu'à 1000 ou $1100 \mathrm{~m}$. Beaucoup plus rares, les stations à $P$. nigra ne fournissent pas ici une image de l'extension de cette espèce. Lascombe (1974) la rencontre entre 160 et $300 \mathrm{~m}$.

- e. Choisi parmi les 4 stations à Dugesia tigrina connues dans la région au départ de l'étude (Russier 1971), l'étang des Bruyères abrite une population nombreuse de cette espèce qui étend régulièrement le domaine où elle est signalée. On doit maintenant la considérer comme sporadiquement présente de $1100 \mathrm{~m}$ (lacs du Puy-de-Dôme, selon le même auteur) jusqu'au niveau du Rhône, dans les bras morts duquel elle abonde.

La question se pose donc, à ce stade, de savoir dans quelle mesure de régime thermique de l'eau justifie la zonation altitudinale et les recouvrements dans cette zonation, là où ils se manifestent.

\subsection{La zonation thermique}

La figure 1 reproduit la situation qui a régné pendant l'année 1973 ainsi que la traduit la méthode de l'inversion du saccharose, précise à $\pm 0,2^{\circ} \mathrm{C}$ (Pattee, 1978). La considération des décimales dans la discussion se justifiera par le fait qu'il s'agit toujours des moyennes de plusieurs valeurs originales.

La zonation thermique des 3 espèces rhéophiles ressort assez nettement, mais avec un certain recouvrement (de $0,5^{\circ} \mathrm{C}$ entre $C$. alpina et $F$. felina, de $1,2^{\circ} \mathrm{C}$ entre $P$. felina et $D$. gonocephala). Comme pour la distribution en fonction de l'altitude, $P$. tenuis et nigra apparais-

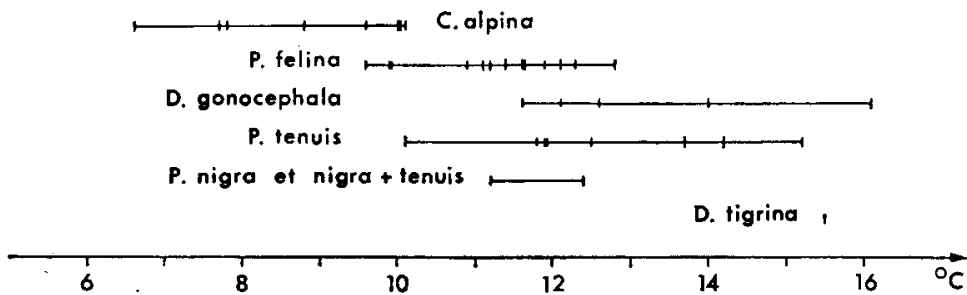

Fig. 1. - Températures moyennes de l'année 1973 dans les stations occupées par les différentes espèces: moyennes "exponentielles " enregistrées par la méthode de l'inversion du saccharose. 
sent parallèlement aux deux espèces précédentes. Enfin, $D$. tigrina vit, dans l'étang des Bruyères, à une température supérieure aux autres.

La figure 2 décrit, avec les restrictions énoncées au paragraphe Stations et Méthode, une situation plus générale, de 1971 à 1973 ou 1974, avec indication des valeurs relatives aux saisons extrêmes hiver et été et des moyennes annuelles arithmétiques et "exponentielles ». Tous ces paramètres font apparaître, de façon plus ou moins tranchée, les mêmes phénomènes que précédemment. La zonation des 3 espèces rhéophiles apparaît le plus nettement dans la moyenne annuelle exponentielle: $C$. alpina se rencontre entre 6,8 et $9,9^{\circ} \mathrm{C}, P$. felina entre 9,4 et $12,3^{\circ} \mathrm{C}, D$. gonocephala entre 12,0 et $15,0^{\circ} \mathrm{C}$. Les recouvrements sont donc respectivement 0,5 et $0,3^{\circ} \mathrm{C}$.

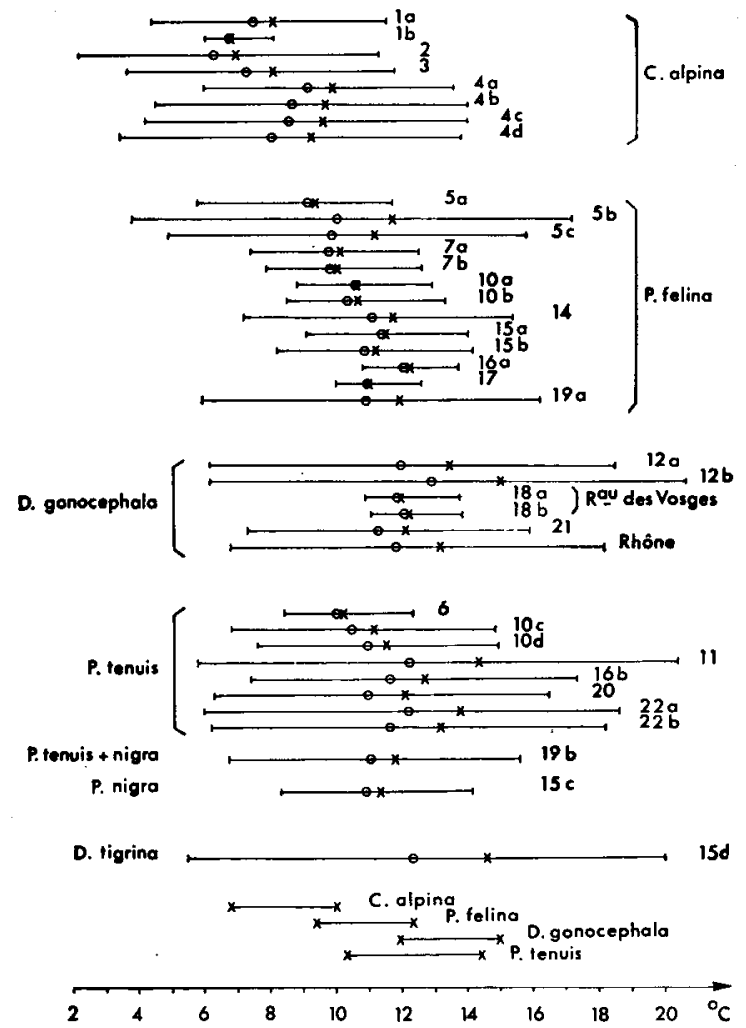

Fig. 2. - Températures moyennes établies d'après les 4 années 1971 à 1974 dans les stations référencées à droite.

Limile gauche des segments: moyenne des hivers.

Limite droite des segments: moyenne des étés.

Cercle: moyenne arithmétique des 4 saisons.

$\mathrm{X}$ : moyenne "exponentielle " des 4 saisons.

En bas sont récapitulées les gammes des températures annuelles (moyennes " exponentielles") où se rencontent les 4 espèces principales. 
Entre les deux premières zones, ces recouvrements sont le fait des slations suivantes :

- la station à $C$. alpina la plus chaude $\left(9,9^{\circ} \mathrm{C}\right)$, représentée par la source de Corcelles (4a). La température moyenne de cette eau se ı efroidit progressivement jusqu'à $9,3^{\circ} \mathrm{C}$ au cours des $100 \mathrm{~m}$ en aval, par suite de l'abaissement des valeurs hivernales.

- la station à $P$. felina la plus froide $\left(9,4^{\circ} \mathrm{C}\right)$, représentée par la source de l'Yzeron (5a). La température moyenne se réchauffe ensuite notablement jusqu'à $11,8^{\circ} \mathrm{C}$ par suite de l'élévation des valeurs estivales.

Dans l'un et l'autre cas, l'espèce présente dans tout le secteur semble davantage en accord avec les conditions qui règnent à l'aval immédiat de la source que dans le griffon ou le suintement eux-mêmes. Peut-être ces deux sources représentent-elles un milieu trop restreint pour être colonisé ou assurer le maintien de l'espèce opposée ?

Les recouvrements entre les zones à $P$. felina et $D$. gonocephala sont également le fait de deux sources : celles de Frontonas à $12,3^{\circ} \mathrm{C}$ dans le mètre qui suit sa sortie de terre (16a) et celle de Cailloux à $12,0^{\circ} \mathrm{C}$ (18a).

La moyenne annuelle arithmétique reflète une situation analogue, mais avec davantage de recouvrements. La zonation est aussi présente, quoique plus floue, dans les valeurs estivales et même les valeurs hivernales des espèces rhéophiles.

\subsection{Interprétation et discussion}

Il convient tout d'abord de conserver à l'esprit la correction qui s'impose concernant les domaines de $C$. alpina et $D$. tigrina : ceux-ci s'étendent encore à des altitudes nettement plus élevées que les stations prospectées pour l'une et pour l'autre. Ces espèces doivent donc tolérer des températures plus basses que celles enregistrées ici.

Les cliverses Planaires ont des habitats assez tranchés en fonction cie l'agitation de l'eau, les unes étant rhéophiles, les autres limnophiles. Elles sont soumises à des microclimats locaux dus à l'exposition, soit directement, soit indirectement par l'origine de l'eau; les écarts qui en résultent entre moyennes annuelles peuvent atteindre $1,5^{\circ} \mathrm{C}$ (Pattee 1979). La constatation la plus évidente que nous venons cic faire est cependant la coïncidence entre zonation altitudinale et zonation thermique.

C. alpina règne seule en altitude et aux températures annuelles basses, dont la linite inférieure n'est pas déterminée ici. Lui succèdent, avec peu de recouvrement, $P$. felina puis $D$. gonocephala. Cette dernière tolère, dans le Rhône et dans l'Amby (stations $12 \mathrm{a}$ et $12 \mathrm{~b}$ ) 
des moyennes estivales de 18 à $20^{\circ} \mathrm{C}$. Les différences entre les espèces jumelles $P$. nigra et $P$. tenuis sont trop subtiles (Lascombe 1974) pour apparaître au niveau de cette étude. On constate seulement que ce groupe de Polycelis limnophiles occupe une zone altitudinale et thermique recouvrant celles des deux dernières espèces rhéophiles.

Toutes sont installées depuis longtemps dans la région et on peut considérer qu'elles ont atteint leur extension définitive dans les conditions actuelles. La moyenne annuelle la plus élevée trouvée pour $P$. tenuis est $14,4^{\circ} \mathrm{C}$ dans un étang ensoleillé. Immigrante américaine, D. tigrina est apparue en Europe en 1925 et en France en 1946 (Russier et Lascombe 1970). Dans les serres et les aquariums tropicaux, dans l'étang des Bruyères avec la moyenne annuelle de $14,6^{\circ} \mathrm{C}$, il semble qu'elle colonise d'abord une niche à l'extrémité du domaine des Polycelis. La référence aux autres stations à $D$. tigrina signalées dans la littérature permet cependant d'affirmer que cette espèce se propage actuellement aux mêmes températures que ses parentes limnophiles. Sa niche est seulement plus large de ce point de vue. La comparaison mériterait d'ailleurs d'être entreprise avec les thermophiles Dugesia polychroa et $D$. lugubris, autres formes jumelles indigènes.

La question se pose ensuite de savoir quel est le paramère thermique susceptible de faire apparaître la meilleure zonation de ces espèces et donc de justifier cette dernière. La température maximale instantanée atteinte pour l'année ne donne pas, nous l'avons vu, de résultats satisfaisants. Le tableau suivant où l'on compare le biotope à $C$. alpina de Corcelles (4c) et celui à D. gonocephala du Rhône en fournit un exemple supplémentaire :

\begin{tabular}{lccc}
\hline & $\begin{array}{l}\text { Maximum } \\
\text { instantané }\end{array}$ & $\begin{array}{l}\text { Moyenne } \\
\text { estivale }\end{array}$ & $\begin{array}{c}\text { Moyenne } \\
\text { annuelle }\end{array}$ \\
\hline Corcelles, C. alpina & $25^{\circ} \mathrm{C} 1$ & $14,0^{\circ} \mathrm{C}$ & $9,6^{\circ} \mathrm{C}$ \\
Rhône, D. gonocephala & $22^{\circ} \mathrm{C} 1$ & $18,2{ }^{\circ} \mathrm{C}^{2}$ & $13,2{ }^{\circ} \mathrm{C} 2$ \\
\hline
\end{tabular}

Les niveaux moyens - y compris le niveau estival - nous apparaissent donc assez indépendants des maximums instantanés et mieux en relation avec la succession des espèces.

Les températures de l'été, saison où les populations aquatiques sont très actives, pourraient jouer un rôle écologique déterminant. La fig. 2 montre que la discrimination entre espèces rhéophiles basée sur ce paramètre est assez mauvaise. Les limites de $C$. alpina sont de

2. Selon Persat (1976). 
8,1 et $14,0^{\circ} \mathrm{C}$, celles de $P$. felina de 11,7 et $17,2^{\circ} \mathrm{C}$, celles de $D$. gonocephala de 13,7 et $20,6^{\circ} \mathrm{C}$. Les recouvrements sont considérables. $\mathrm{C}^{\prime}$ est bien la moyenne annuelle "exponentielle " calculée d'après la loi de Van't Hoff et sur plusieurs années successives qui justifie de la façon la plus satisfaisante la succession de espèces rhéophiles.

Cette constatation, ainsi que les élevages au laboratoire (Pattee, Lascombe et Delolme 1973) permettent d'exclure un rôle limitant, directement ou indirectement létal, de la température. Un rapprochement s'impose donc avec les phénomènes commandés par le métabolisme de l'animal, lui-même plus ou moins étroitement soumis à la loi cles réactions chimiques de Van't Hoff. On pense, en particulier, à l'activité locomotrice et par suite alimentaire, à la croissance, à la fécondité. Ces deux dernières sont les composantes du taux intrinsèque d'accroissement naturel des populations, c'est-à-dire de leur capacité maximale de multiplication dans un milieu aux conditions par ailleurs optimales. La fig. 3 montre que la coïncidence avec ce dernier para-

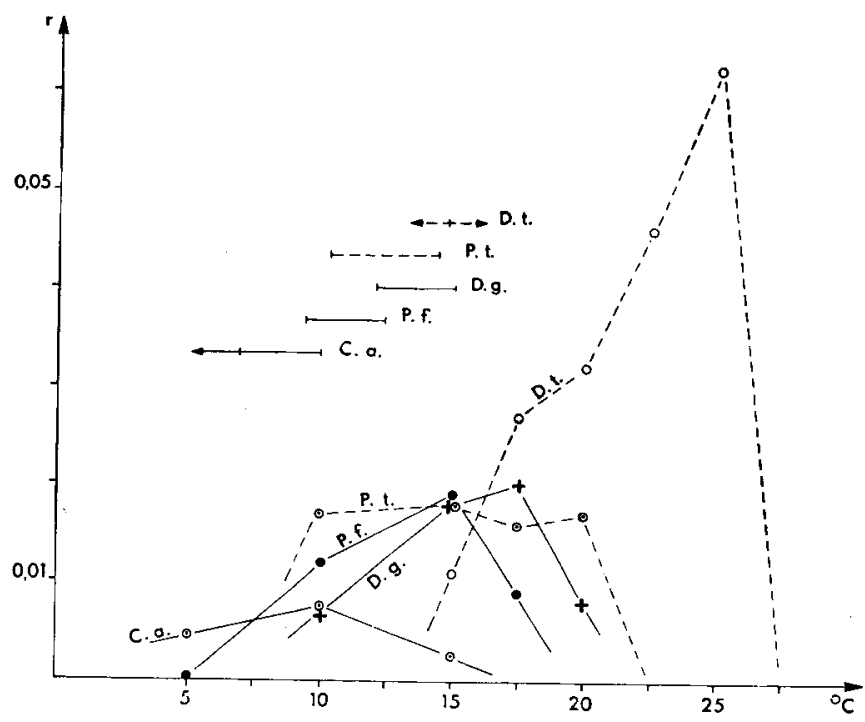

Fig. 3. - Comparaison entre habitat et capacité reproductive des 5 espèces de Planaires.

D. t. = Dugesia tigrina

P. t. = Polycelis tenuis

D. g. = Dugesia gonocephala

P. f. = Polycelis felina

C. a. = Crenobia alpina .

En haut: gamme des températures moyennes annuelles mises en évidence sur le terrain, d'après la figure 2 .

En bas: effet de la température sur le taux intrinsèque d'accroissement naturel $r$ de populations des 5 espèces, d'après les travaux antérieurs (Pattee 1970 et 1972, Russier-Delolme 1972).

En traits pleins: tracés relatifs aux espèces rhéophiles.

En traits interrompus: tracés relatifs aux espèces limnophiles. 
mètre n'est pas parfaite en ce qui concerne les valeurs absolues de la température, mais que les dispositions relatives sont les mêmes.

L'activité, la croissance et la fécondité conditionnent l'efficacité dans l'exploitation du milieu et la possibilité de priver les autres espèces des mêmes ressources. A ce stade, l'étude apporte donc un argument supplémentaire à l'hypothèse d'une compétition interspécifique entre les trois Planaires rhéophiles, compétition dont l'issue serait céterminée directement par le niveau moyen de la température. Mais, comme l'ont si justement montré Reynoldson et Bellamy en 1970, coïncidence ne veut pas dire causalité : la démonstration rigoureuse d'une compétition exige des manipulations de populations et de ressources sur le terrain.

Une commande indirecte de la zonation des Planaires rhéophiles par celle de leurs proies semble en tous cas exclue: Gammares et larves d'Insectes variées abondent dans les 3 zones considérées. Lock et Reynoldson (1976) ont montré que $C$. alpina et $P$. felina s'en nourrissent.

La distribution de $P$. tenuis s'explique de la façon suivante : elle est limitée à la fois par les températures basses et par l'agitation de l'eau: d'une part, sa reproduction cesse en-dessous de $10^{\circ} \mathrm{C}$ (fig. 3), dans la mesure où le réchauffement estival à partir de ce niveau moyen n'est pas suffisamment long ou intense pour stimuler la ponte; d'autre part, l'agitation de l'eau la décroche du substrat (Pattee et Bournaud 1970). Elle laisse donc place libre, aussi bien à $C$. alpina dans les milieux lénitiques froids, qu'aux espèces rhéophiles en milieu lotique. Par contre, sa plus grande efficacité d'accroissement (fig. 3) et d'alimentation (Reynoldson et Bellamy 1973) lui permettraient d'éliminer les espèces précédentes dans tout milieu lénitique où elle peut se maintenir. Cette hypothèse, avancée par Pattee en 1972 trouve une confirmation dans les enregistrements présentés ici.

Ses relations avec $D$. tigrina sont plus difficiles à préciser. S'agit-il a'une limitation de la première ou d'un défaut de compétitivité aux températures élevées, ou d'une spécialisation alimentaire du même ordre que celles mises en évidence par Reynoldson (1966) entre espèces limnophiles indigènes, ces mécanismes agissant séparément ou conjointement? D'autres études sont nécessaires sur ce point.

Quant au fait que la zonation thermique des Planaires rhéophiles soit plus nette selon les résultals moyens des 4 années que pour la seule année 1973, si ce résultat n'est pas dû au hasard, il signifierait que la température exerce ses effets de façon indirecte et lente, au rythme des générations successives, des colonisations et recolonisations éventuelles de stations - le temps de génération des Planaires à reproduction sexuée est de 100 à 600 jours selon l'espèce et la température; on conçoit qu'un équilibre donné soit fondé sur plusieurs années successives. 


\subsection{Vérification de l'une des hypothèses avancées}

A l'époque de l'étude, le ruisseau des Vosges (station 18) n'abritait, en fait de Planaires, que Dugesia gonocephala et Dendrocoelum lacteum, toutes deux en grand nombre. Il apparaissait comme la plus froide des stations à $D$. gonocephala, sa température moyenne étant identique, voir inférieure à celle de certaines stations à $P$. felina.

Lorsque ce résultat devint évident, à l'été 1975, une centaine d'individus de cette dernière espèce, prélevés aux sources de l'Yzeron (station 5), furent déversés dans le ruisseau des Vosges, qui n'en contenait aucun. N'ayant pas retrouvé les individus déversés ou leurs descendants en 1976, j'avais conclu un peu prématurément à l'échec de cette introduction et cessé les recherches. C'est seulement à l'automne 1978 que des collègues m'ont fortuitement alerté sur la présence de $P$. felina clans le ruisseau. La prospection qui a suivi démontre le remplacement total de $D$. gonocephala par une population nombreuse de P. felina. D. lacteum a conservé les mêmes effectifs qu'auparavant.

Ce remplacement d'une espèce par une autre réduit le recouvrement entre les zones à $P$. felina et à $D$. gonocephala, tel qu'il apparaît sur les figures 1 et 2 . S'il n'est pas fortuit, ce qui paraît malgré tout peu probable, il montre ou confirme :

- la compétition interspécifique qui règne très probablement entre ces deux espèces.

- la supériorité de $P$. felina à $10^{\circ} \mathrm{C}$ et la validité des mesures de laboratoire qui conduisent aux mêmes conclusions (fig. 3).

- le mécanisme de l'action de la température par l'intermédiaire de cette compétition dont elle gouverne l'issue.

- la lenteur des phénomènes en cause. Bien que le temps de génération de $P$. felina (race asexuée) soit de 70 jours environ à $10^{\circ} \mathrm{C}$ (Pattee 1969), plusieurs années ont été nécessaires pour que se manifeste un résultat sur un secteur d'une cinquantaine de $\mathrm{m}^{2}$.

- les barrières qui s'opposent à la dispersion de la faune aquatique. $P$. felina existait déjà à $500 \mathrm{~m}$ de là dans la même vallée (à la station 19 a). Mais elle était séparée du secteur à $D$. gonocephala par un fossé marécageux à $P$. tenuis et $P$. nigra et une zone plus courante en aval de l'affluent, puis par quelques centaines de mètres et de petites cascades qu'elle aurait eu à remonter sur le cours principal.

\section{TRAVAUX CITÉS}

Al.AuSE (P.). 1968. - Contribution à l'écologie des Planaires d'eau courante. Observations en Languedoc méditerranéen. Le facteur acidité carbonique. Thèse, Montpellier, I : 550 p. et II : 179 p. 
ARNDr (W.). 1922. - Untersuchungen an Bachtricladen. Zs. wiss. Zool., 120 : 98 146.

BALI. (I. R.). 1964. - Preliminary observations on the freshwater Tricladida of North Hertfordshire. Trans. Herts. nat. Hist. Soc. Fld Club, $26: 37-43$.

Bfauchamp (R.S. A.) et Ullyott (P.). 1932. - Competitive relationships between certain species of freshwater Triclads. J. Ecol., $20: 200-208$.

BECKER (E.). 1960. - Zur geographischen Verbreitung und ökologie der tricladen Turbellarien in Württemberg. Jh. Ver. vaterl. Naturk. Wiirtt., $115: 267-305$.

BrRThet (P.). 1960. - La mesure écologique de la température par détermination de la vitesse d'inversion du saccharose. Vegetatio, $9:$ 197-207.

BRown (L. A.). 1929. - The natural history of Cladocerans in relation to temperature. I. Distribution and temperature limits for vital activities. Amer. Natur., $63: 248-264$.

CARPenter (K.). 1928. - On the distribution of freshwater Turbellaria in the Aberystwyth district, with especial reference to two icc-age relicts. J. Ecol., $16: 105-122$.

CInNIIIER (C. M.). 1965. - Environmental factors affecting the local distribution and abundance of four species of stream-dwelling Triclads. Ph. D. Thesis, Indiana Univ., $104 \mathrm{p}$.

DahM (A. G.). - 1958. - Taxonomy and ecology of five species groups in the family Planariidae (Turbellaria Tricladia Paludicola). Malmö, $241 \mathrm{p}$.

Décamps (H.). 1967. - Ecologie des Trichoptères de la vallée d'Aure (HautesPyrénées). Annls Limnol., 3 : 399-577.

Décamps (H.). 1968. - Vicariances écologiques chez les Trichoptères des Pyrénées. Annls Limnol., 4: 1-50.

Despax (R.). 1927. - Observations biologiques en montagne. Recherches sur les facteurs de la distribution des organismes. Bull. biol., 61: 126-142.

Donds (G. S.) et HISAW (F. L.). 1925. - Ecological studies on aquatic insects. IV. Altitudinal range and zonation of Mayflies, Stoneflies and Caddisflies in the Colorado Rockies. Ecology, 6: 380-390.

Eningron (J. M.) et HildRew (A. H.). 1973. - Experimental observations relating to the distribution of net-spinning Trichoptera in streams. Verh. internat. Verein. Limnol., 18: 1549-1558.

FlössNi:R (D.). 1963. - Zur ökologie der Bergbachtrikladen im Erzgebirge. Limnologica Berlin, $1: 35-44$.

Giesen-HildebRand (D.). 1975. - Die Planarienfauna der Siebengebirgsbäche. Eine ökologisch-historische Untersuchung unter besonderer Berücksichtigung der Befunde von Voigt (1894). Decheniana, $128: 21-29$.

HuLt (M.), 1949. - Aperçu des relations entre la pente et les relations piscicoles des eaux courantes. Rev. suisse Hydrol., 11 : 332-351.

KAMLER (E.). 1965. - Thermal conditions in mountain waters and their influence on the distribution of Plecoptera and Ephemeroptera larvae. Ekol. pol., Ser. A, $13: 377-414$.

Kı⿻上Katsu (M.) 1965. - On the ecology and distribution of freshwater Planarians in the Japanese Islands, with special reference to their vertical distribution. Hydrobiologia, $26: 349-408$.

KawaknTSU (M.). 1970. - Further studies on the vertical distribution of freshwater Planarians in the Japanese Islands. American Zoologist, $10: 527$.

KAWAKATSU (M.). 1974. - Chapter 14. Further studies on the vertical distribution of freshwater Planarians in the Japanese Islands. 291-338. In RISER (N.W.) and MorSE (M. P.) Biology of the Turbellaria. New York.

Kawakatsu (M.), Nimura (F.) et Horikoshi (I.). 1974. - Report on the ecological survey of freshwater Planarians in the Saku Basin and its adjacent mountainous districts, Honshû. Zool. Mag. zool. Soc. Japan, 83 : 225-230. 
LAsCOMBBE (C.). 1974. - Recherches écologiques et biogéographiques sur deux espèces jumelles de Planaires d'eau douce de la région lyonnaise. Bull. mens. Soc. linn. Lyon, 43 : $168-176$ et $189-215$.

L.joter (P.). 1969. - Etude écologique des Planaires Triclades d'eau douce en Provence occidentale. Thèse Spécialité, Marseille, $162 \mathrm{p}$.

LegII:R (P.). 1972. - Répartition et étagement de l'habitat chez les Planaires Triclades d'eau douce en Provence occidentale. Annls Limnol., 8 : 31-40.

Leloup (E.). 1944. - Recherches sur les Triclades dulcicoles épigés de la forêt de Soignes. Mém. Mus. r. Hist. nat. Belg., 102 : 1-112.

Lock (M. A.) et ReYnoldson (T. B.). 1976. - The role of interspecific competition in the distribution of two stream-dwelling Triclads, Crenobia alpina (Dana) and Polycelis felina (Dalyell) in North Wales. J. Anim. Ecol., 45 : 581-592.

LuTHER (A.). 1961. - Die Turbellarien Ostfennoskandien. II. Tricladia. Fauna fenn., $11: 1-42$.

Macan (T. T.). 1956. - Influence of temperature on freshwater animals. Discovery, $17: 275-277$.

MACAN (T. T.). 1961. - Factors that limit the range of freshwater animals. Biol. Rev., $36: 151-198$.

Madsen (B. L.). 1963, - Økologiske undersøgelser af nogle østjyske vandløb. II. Planarier og igler. Flora og Fauna, $69: 113-125$.

Mercier (L.). 1909. - Sur la présence de Planaria alpina Dana aux environs de Nancy. Arch. Zool. exp. gén. (5) I : XLIX-LVII.

MooRI: (J.A.). 1939. - Temperature tolerance and rates of development in the eggs of Amphibia. Ecology, 20 : 459-478.

MooRE (J.A.), 1949. - Geographic variation of adaptative character in Rana pipiens Schreber. Evolution, $3: 1-24$.

Oye (E. L.) van. 1941. - Verbreitung und ökologie der paludicolen Tricladen in Belgien. Arch. Hydrobiol., 38 : 110-147.

PARK (T.). 1945. - A further report on toleration experiments by ecology classes. Ecology, 26 : 305-308.

PatTee (E.). 1965. - Sténothermie et eurythermie. Les Invertébrés d'eau douce et la variation journalière de température. Annls Limnol., 1 : 281-434.

Pattre (E.). 1966. - Coefficients therminques et écologie de quelques Planaires d'eau douce. 1. Tolérance des adultes. Annls Limnol., 2 : 469-475.

Pattre (E.). 1968. - Coefficients thermiques et écologie de quelques Planaires d'eau douce. 2. Tolérance de Dugesia gonocephala. Annls Limnol, $4: 99$. 104.

Patrie (E.). 1969 a. - Coefficients thermiques et écologie de quelques Planaires d'eau douce. 3. La reproduction des deux espèces montagnardes. Annls Limnol., 5 : 9-24.

Pattee (E.). 1969 b. - Contribution expérimentale à l'écologie de la Planaire alpine, Crenobia alpina (Dana). Bull. Soc. zool. Fr., 94 : 269-276.

Pattee (E.). 1970. - Coefficients thermiques et écologie de quelques Planaires d'eau douce. 4. La reproduction de Dugesia gonocephala. Annls Limnol. 6 : 293-304.

Pattee (E.). 1972. - Coefficients thermiques et écologie de quelques Planaires d'eau douce. 5. La reproduction des espèces jumelles Polycelis nigra et Polycelis tenuis. Annls Limnol., 8 : 11-30.

Patree (E.). 1978. - Un indice thermique utile en écologie. 1. La mesure de la température par la méthode de l'inversion du saccharose. Bull. Ecol., 9: 253258.

Patrie (E.). 1979. - Un indice thermique utile en écologie. 2. Quelques valeurs annuelles et trimestrielles obtenues en eau douce par la méthode de l'inversion du saccharose. Bull. Ecol. (sous presse). 
Patree (E.) et Bournaud (M.). 1970. - Etude expérimentale de la rhéophilie chez des Planaires Triclades d'eau courante. Schweiz. Z. Hydrol., 32: 181191.

Pattee (E.), Lascombe (C.) et Delolme (R.). 1973. - Effects of temperature on the distribution of Turbellarian Triclads. 201-207. In WIESER (W.) Effects of temperature on ectothermic organisms, Berlin.

Persat (H.). 1976. - Principaux aspects de l'écologie de l'Ombre commun, Thymallus thymallus (L. 1758) (Poissons Salmonidés). Thèse spécialité, Lyon, $115 \mathrm{p}$.

Philipson (G. N.) et Moorhouse (B. H. S.). 1976. - Respiratory behaviour of larvae of four species of the family Polycentropodidae (Trichoptera). Freshwater Biol., 6 : 347-353.

Pleskot (G.). 1951. - Wassertemperatur und Leben im Bach. Wett. u. Leben, $3: 129-143$.

ReYNoldson (T. B.). 1960. - A quantitative study of the population biology of Polycelis tenuis (Ijima) (Turbellaria, Tricladia). Oikos, 11 : 125-141.

Reynoldson (T. B.). 1966. - The distribution and abundance of lake-dwelling Triclads-towards a hypothesis. Adv. ecol. Res., 3: 1-71.

RFynoldson (T. B.) et Bellamy (L. S.). 1970. - The establishment of interspecific competition in field populations, with an example of competition in action between Polycelis nigra (Müll.) and P. tenuis (Ijima) (Turbellaria, Tricladida). Proc. Adv. Study Inst. Dynamics Numbers Popul. (Oosterbeek), 28229 i.

Reynordson (T. B.) et Bellamy (L. S.). 1973. - Interspecific competition in lakedwelling Triclads. A laboratory study. Oikos, 24 : 301-313.

Roberts (J. L.). 1957. - Thermal acclimation of metabolism in the crab, Pachy. grapsus crassipes Randall. II. Mechanisms and the influence of season and latitude. Physiol. Zoöl., $30:$ 242-255.

Roux (C.) et Rovx (A. L.). 1967. - Température et métabolisme respiratoire d'espèces sympatriques de Gammares du groupe pulex (Crustacés, Amphipodes). Annls Limnol., 3 : 3-16.

Russier (R.). 1971. - La Planaire américaine Dugesia tigrina dans la région lyonnaise: essai d'explication écophysiologique de son implantation. Thèse Spécialité, Lyon, 93 p.

Russiar-Delolme (R.). 1972. - Coefficients thermiques et écologie de quelques Planaires d'eau douce. 6. Dugesia tigrina. Annls Limnol., 8 : 119-140.

Russier (R.) et Lascombe (C.). 1970. - La Planaire américaine Dugesia tigrina dans la région lyonnaise: écologie et tolérance thermique. Bull. mens. Soc. limn. Lyon, 39 : 197-206.

SCIILIEPER (C.) et BLäsing (I.). 1952. - Uber Unterschiede in dem individuellen und ökologischen Temperaturbereich von Planaria alpina Dana. Arch. Hydro. biol., 47 : 288-294.

Schoiander (P. F.), Flagg (W.), Walters (V.) et Irving (L.). 1953. - Climatic adaptation in arctic and tropical poikilotherms. Physiol. Zoöl., 26: 67-92.

SPENCE (J. A.) et HyNES (H. B. N.). 1971. - Differences in fish populations upstream and downstream of a mainstream impoundment. J. Fish. Res. Board Canada, $28: 45-46$.

Stankovic (S.). 1935. - Uber die Verbreitung und ökologie der Quellentricladen auf der Balkanhalbinsel. Zoogeographica, 2 : 147-203.

STEINßöck (O.). 1942 a. - Das Verhalten von Planaria alpina Dana in der Natur und im Laboratoriumsversuch. Mem. Ist. ital. Idrobiol. de Marchi, 1 : 63-75.

STrinböck (O.). 1942 b. - Eigenheiten boreoalpiner Tierverbreitung. Mem. Ist. ital. Idrobiol. de Marchi, 1 : 129-143.

STEINBöcK (O.). 1951. - Turbellarienstudien am Lago Maggiore. Mem. Ist. ital. Idrobiol. de Marchi, 6: 137-164 et 165-176. 
Stcinmann (P.). 1907. - Geographisches und Biologisches von Gebirgsbachplanarien. Arch. Hydrobiol., 2 : 186-217.

TASIIIAN (R. E.). 1956. - Geographic variation in the respiratory metabolism and temperature coefficient in tropical and temperate forms of the fiddler crab, Uca pugnax. Zoologica, $41: 39-47$.

Tiuinemann (A.). 1913. - Der Bergbach des Sauerlandes. Kurze Zusammenfassung der Ergebnisse faunistisch-biologischer Untersuchungen. Arch. Hydrobiol., $8: 432-445$.

VaIllant (F.). 1967 a. - Sur le choix des espèces indicatrices pour une zonation des eaux courantes. Trav. Lab. Hydrobiol. Grenoble, 57-58 : 7-15.

VaIll.ant (F.). 1967 b. - La répartition des Wiedemannia dans les cours d'eau et leur utilisation comme indicateur de zones écologiques (Diptera Empididae). Annls Limnol., 3 : 267-293.

VAtL.IANT (F.). 1972. - Une zonation thermique des cours d'eau alpestres basée sur la répartition de quelques invertébrés. Trav. Lab. Hydrobiol. Grenoble, 63 : $177-188$.

VANbel (A.). 1919. - Contribution à la connaissance de la faune des eaux douces du Jura. Bull. Soc. zool. Fr., 44 : 80-94.

VernkerG (F.J.). 1959 a. - Studies on the physiological variation between tropical and temperate zone fiddler crabs of the genus Uca. 2. Oxygen consumption of whole organisms. Biol. Bull., 117 : 163-184.

VisRnberg (F. J.). 1959 b. - Studies on the physiological variation between tropical and temperate zone fiddler crabs of the genus $U c a$. 3. The influence of temperature acclimation on oxygen consumption of whole organisms. Biol. Bull., 117 : 582-593.

Vernierg (F. J.) et Tashian (R. E.). 1959. - Studies on the physiological variation between tropical and temperate zone fiddler crabs of the genus Uca. 1 . Thermal death limits. Ecology, $40: 589-593$.

Verneatux (J.). 1976. - Biotypologie de l'écosystème "eau courante". La structure biotypologique. C. R. Acad. Sci., Paris, sér. D, 283 : 1663-1666.

Vernenux (J.). 1977. - Biotypologie de l'écosystème "eau courante ". Déterminisme approché de la structure biotypologique. C. R. Acad. Sci. Paris, sér. D, $284:$ :77-79.

VoJGr (W.). 1895. - Planaria gonocephala als Eindringling in das Verbreitungsgebiet von Planaria alpina und Polycelis cornuta. Zool. Jb., Abt. Syst. Oekol. Geogr., 8: 131-176.

Watsur (B. M.). 1948. - The oxygen requirements and thermal resistance of chironomid larvae from flowing and from still water. J. exp. Biol., $25: 35-44$.

WARI) (J.V.). 1974. - A temperature-stressed ecosystem below a hypolimnial release mountain reservoir. Arch. Hydrobiol., 74 : 247-275.

WhitneY (R. J.). 1939. - The thermal resistance of Mayfly nymphs from ponds and streams. J. exp. Biol., $16: 374-385$.

Wrigut (J. F.). 1972. - The distribution of stream-dwelling Triclads (Platyhelminthes) in North Wales. Freswater Biol., 2: 57-64. 